\title{
New interpolation spaces and strict Hölder regularity for fractional abstract Cauchy problem
}

\author{
Md Mansur Alam', Shruti Dubey ${ }^{1 *}$ (D) and Dumitru Baleanu' 2,3,4
}

\section{"Correspondence:}

sdubey@iitm.ac.in

'Department of Mathematics, IIT

Madras, Chennai, India

Full list of author information is

available at the end of the article

\begin{abstract}
We know that interpolation spaces in terms of analytic semigroup have a significant role into the study of strict Hölder regularity of solutions of classical abstract Cauchy problem (ACP). In this paper, we first construct interpolation spaces in terms of solution operators in fractional calculus and characterize these spaces. Then we establish strict Hölder regularity of mild solutions of fractional order ACP.
\end{abstract}

MSC: 34A08; 35B65; 35D99; 46B70

Keywords: Interpolation space; Fractional calculus; Hölder continuity; Strict solution; Solution operator

\section{Introduction}

Let $(X,\|\cdot\|)$ be a Banach space. First, we take into consideration of the following abstract Cauchy problem:

$$
\left\{\begin{array}{l}
\frac{d v}{d t}=A v(t)+\mathfrak{F}(t), \quad 0<t \leq T, \\
v(0)=v_{0}
\end{array}\right.
$$

where $\mathfrak{F}:[0, T] \rightarrow X, v_{0} \in X$, and $A: D(A) \subset X \rightarrow X$ is densely defined operator satisfying following conditions:

(i) $\sum_{\theta}:=\{\mu \in \mathbb{C}: \mu \neq 0,|\arg (\mu)|<\theta\} \subset \rho(A)$,

(ii) $\left\|(\mu I-A)^{-1}\right\|_{L(X)} \leq \frac{N}{|\mu|}, \forall \mu \in \sum_{\theta}$,

for some $\theta \in\left(\frac{\pi}{2}, \pi\right), N>0$. The sets $\rho(A), L(X)$ are denoted as resolvent set of $A$ and the set of all bounded linear operators on $X$, respectively. Such operator $A$, is known as sectorial operator, and it generates the analytic semigroup $\{T(t)\}_{t \geq 0}$ on $X$ (cf. [1]). The study of strict solution of (1.1) is very well known. For instance, we refer to [1, Chap. 4], [2] and the references therein. In these cited papers, the authors proved strict Hölder regularity of the mild solution of (1.1) under suitable space and time regularity of the initial datum. More precisely, if $\mathfrak{F} \in C^{\theta}([0, T] ; X)$, where $0<\theta<1, v_{0} \in D(A)$ with $A v_{0}+\mathfrak{F}(0) \in D_{A}(\theta, \infty)$, an interpolation space, then (1.1) has strict solution. The results have been obtained by

(c) The Author(s) 2021. This article is licensed under a Creative Commons Attribution 4.0 International License, which permits use, sharing, adaptation, distribution and reproduction in any medium or format, as long as you give appropriate credit to the original author(s) and the source, provide a link to the Creative Commons licence, and indicate if changes were made. The images or other third party material in this article are included in the article's Creative Commons licence, unless indicated otherwise in a credit line to the material. If material is not included in the article's Creative Commons licence and your intended use is not permitted by statutory regulation or exceeds the permitted use, you will need to obtain permission directly from the copyright holder. To view a copy of this licence, visit http://creativecommons.org/licenses/by/4.0/. 
using the advantage of representation of interpolation space $D_{A}(\theta, \infty)$ in terms of analytic semigroup. Here we are interested in the investigation of similar results for the following fractional order ACP on $X$ :

$$
\left\{\begin{array}{l}
{ }^{c} D_{t}^{\alpha} v(t)=A v(t)+\mathfrak{F}(t), \quad 0<t \leq T, \\
v(0)=v_{0}
\end{array}\right.
$$

where ${ }^{c} D_{t}^{\alpha}$ represents Caputo fractional differential operator of order $\alpha \in(0,1)$.

Over the last few decades, the study in the area of fractional calculus has influenced the researchers owing to its generous applicability in the branch of science and engineering. Specifically, we refer the work [3] for some substantial applications of fractional differential equations.

There have been intensive investigation on existence, uniqueness and regularity of solution for variety of generalized model of (1.2). For the enthusiastic reader, we refer to [4-6] for linear autonomous case, [7, 8] for semilinear autonomous case, [9] and some work cited therein for the non-autonomous case with delay. But the study of strict regularity of the problem (1.2) is very rare for the case $\alpha \in(0,1)$. Bazhlekova [10], in her precious thesis, studied strict $L^{p}$ - regularity by introducing the concept of solution operators and using the resolvent representation of classical interpolation space $D_{A}(\theta, p)$ for $1<p<\infty$. The case $p=\infty$ (i.e., strict Hölder regularity case) has been studied first by Ph. Clément et al. in their pioneer work [11]. Using some suitable transformation, the author split the problem (1.2) into two abstract problems, one is having homogeneous force function (i.e., $\mathfrak{F}=0$ ) but with inhomogeneous initial data, and another is having inhomogeneous force function $\mathfrak{F}(t)-\mathfrak{F}(0)$ but with homogeneous initial data. The author used method of sum to investigate the regularity of the latter one, in which the problem is converted to an operator equation of the form $B u+C u=v$ on the space $\tilde{X}=\{v \in C([0, T] ; X): v(0)=0\}$ and applied a suitable theorem [11, Theorem 8]. Whereas, the regularity of the first one is solved by exploiting resolvent representation of the interpolation space $D_{A}(\theta, \infty)$. Finally, combining the both results, the author obtained the strict Hölder regularity of the problem (1.2). Also, the work of $\mathrm{Li}$ liu et al. [12] is devoted to examining maximal regularity property of the weighted Hölder space $C_{0}^{\theta}([0, T] ; X)$ for the problem (1.2) by utilizing the concept of $\alpha$-times resolvent families introduced in [13], when $v(0)=v_{0} \in D(A)$.

In contrast to this above-mentioned work, for the first time we are going to prove that the classical real interpolation space $D_{A}(\theta, p)$ can also be represented in terms of $S_{\alpha}(t), T_{\alpha}(t)$, named as "solution operators" of fractional ACP. Guswanto, in [14], explicitly introduced these pair of operators on $X$, defined as follows:

$$
\begin{aligned}
& S_{\alpha}(t)=\frac{1}{2 \pi i} \int_{\Upsilon} e^{\lambda t} \lambda^{\alpha-1}\left(\lambda^{\alpha}-A\right)^{-1} d \lambda, \quad t>0, \\
& T_{\alpha}(t)=\frac{1}{2 \pi i} \int_{\Upsilon} e^{\lambda t}\left(\lambda^{\alpha}-A\right)^{-1} d \lambda, \quad t>0,
\end{aligned}
$$

where $\Upsilon \subset \rho(A)$, an anticlockwise oriented path. He also demonstrated the topological properties of these operators which are somewhat similar to those in classical case; see [15]. These similarities create a great advantage to study the existence, uniqueness and regularity properties of solution of (1.2) in the same fashion as of classical case. However, 
there are some disadvantages as these families do not satisfy semigroup properties and the operator $T_{\alpha}(t)$ has singularity near $t=0$.

On the other hand, the study of strict solutions come into effect when one wants to get the differentiability of the solutions up to $t=0$. In fact, it is known that the $\alpha$-Caputo derivative of classical solutions of (1.2) cannot be extended up to $t=0$, even under the conditions $v_{0} \in D(A), \mathfrak{F} \in C^{\theta}([0, T] ; X)$ (see, e.g., [16]). Thanks to interpolation spaces which play crucial role to fill this gap between classical solutions and strict solutions. It is evident that interpolation spaces can be expressed in terms of semigroup. However, no such expression in terms of $S_{\alpha}(t), T_{\alpha}(t)$ is available in the literature so far. Motivated by this, we analyze the following points:

(I) constructing interpolation spaces in terms of solution operators $S_{\alpha}(t), T_{\alpha}(t)$ of fractional $\mathrm{ACP}$ and characterize these spaces,

(II) establishing the strict Hölder regularity (or maximal Hölder regularity) results for the problem (1.2) utilizing this new representation of interpolation space.

It is worth to mention that this new finding creates not only a great opportunity to study the strict regularity results of the problem (1.2) in the similar fashion as of classical case, but also provide a new sight in the area of fractional calculus.

This paper is organised as follows. In Sect. 2, we present preliminary results on some Hardy-type inequalities, a short introduction of real interpolation spaces and properties of the solution operators. In Sect. 3, we construct interpolation spaces in terms of solution operators and characterize these spaces, which is one of the goals of this paper. In the last section, we establish the sufficient conditions to investigate the strict Hölder regularity of mild solutions of (1.2), and provide an example to illustrate the results.

\section{Some notations, preliminary results and interpolation spaces}

Let $I \subset \mathbb{R}$ be an interval. Traditionally, for a compact interval $I, C(I ; X)$ represents the space of $X$-valued continuous functions on $I$ endowed with the usual supremum norm. The Hölder space $C^{\theta}(I ; X)$ is defined by

$$
C^{\theta}(I ; X):=\left\{g \in C(I ; X):[g]_{\theta}=\sup _{s, t \in I, s \neq t} \frac{\|g(t)-g(s)\|}{|t-s|^{\theta}}<\infty\right\}
$$

assigned with the norm $\|g\|_{C^{\theta}(I ; X)}=\sup _{t \in I}\|g(t)\|+[g]_{\theta}$, where $\theta \in(0,1)$.

The space $L_{*}^{p}(I):=L^{p}\left(I, \frac{d t}{t}\right)$, equipped with norm

$$
\begin{aligned}
& \|g\|_{L_{*}^{p}(I)}=\left(\int_{I}|g(s)|^{p} \frac{d s}{s}\right)^{\frac{1}{p}}, \quad \text { if } 1 \leq p<\infty, \\
& \|g\|_{L_{*}^{\infty}(I)}=\underset{s \in I}{\operatorname{essup} \sup }|g(s)|, \quad \text { if } p=\infty .
\end{aligned}
$$

Definition 2.1 Let $f \in L^{1}([a, b] ; X)$. The operator defined by

$$
\left(I^{\alpha} f\right)(t)=\frac{1}{\Gamma(\alpha)} \int_{a}^{t}(t-s)^{\alpha-1} f(s) d s, \quad \text { a.e } t \in[a, b],
$$

is known as Riemann-Liouville integral of order $\alpha \in(0,1)$. 
For $\alpha>0$, we consider

$$
g_{\alpha}(t)= \begin{cases}\frac{t^{\alpha-1}}{\Gamma(\alpha)}, & t>0 \\ 0, & t \leq 0\end{cases}
$$

The convolution between $f:[0, T] \rightarrow X$ and $g_{\alpha}$ is defined by

$$
\left(g_{\alpha} * f\right)(t)=\frac{1}{\Gamma(\alpha)} \int_{0}^{t}(t-s)^{\alpha-1} f(s) d s, \quad t \in(0, T]
$$

One can verify that, for $\alpha, \beta \in(0,1), g_{\alpha+\beta}=g_{\alpha} * g_{\beta}$.

Definition 2.2 Let $f \in L^{1}([a, b] ; X)$ be such that $I^{1-\alpha}(f(\cdot)-f(a)) \in W^{1,1}([a, b] ; X)$. Then the Caputo derivative of order $\alpha \in(0,1)$ is defined as

$$
\begin{aligned}
{ }^{c} D_{t}^{\alpha} f(t) & =\frac{d}{d t}\left(I^{1-\alpha}(f(\cdot)-f(a))\right)(t) \\
& =\frac{1}{\Gamma(1-\alpha)} \frac{d}{d t} \int_{a}^{t}(t-s)^{-\alpha}(f(s)-f(a)) d s \quad \text { a.e } t \in[a, b] .
\end{aligned}
$$

Definition 2.3 Let $\mathfrak{F} \in L^{1}((0, T) ; X)$. By a mild solution of (1.2), we mean a function $v \in$ $C([0, T] ; X)$ which satisfies the following integral equations:

$$
v(t)=S_{\alpha}(t) v_{0}+\int_{0}^{t} T_{\alpha}(t-s) \mathfrak{F}(s) d s, \quad t \in(0, T] .
$$

Definition 2.4 A function $v \in C([0, T] ; X)$ satisfying:

(i) $v \in C((0, T] ; D(A))$,

(ii) $t \mapsto g_{1-\alpha} *\left(v(\cdot)-v_{0}\right)(t) \in C^{1}((0, T] ; X)$,

(iii) Eq. (1.2),

is called a classical solution of (1.2).

Definition 2.5 A function $v \in C([0, T] ; D(A))$ satisfying:

(i) $t \mapsto g_{1-\alpha} *\left(v(\cdot)-v_{0}\right)(t) \in C^{1}([0, T] ; X)$,

(ii) Eq. (1.2),

is called a strict solution of (1.2).

Lemma 2.6 (Hardy-type inequalities involving Riemann Liouville integral [17]) Let $1<$ $p \leq q<\infty, \frac{1}{p}+\frac{1}{p^{\prime}}=1$, and $u, v$ be non-negative weight functions. Then the boundedness of the linear operator $I^{\alpha}: L^{p}((0, \infty) ; v(t) d t) \rightarrow L^{q}((0, \infty) ; u(t) d t)$ holds if and only if for some $C>0$

$$
\left(\int_{2 R}^{\infty} t^{(\alpha-1) q} u(t) d t\right)^{\frac{1}{q}}\left(\int_{0}^{R}(v(t))^{1-p^{\prime}} d t\right)^{\frac{1}{p^{\prime}}} \leq C \quad \text { for all } R>0
$$

and $\widetilde{I_{\alpha}}: L^{p}((0, \infty) ; v(t) d t) \rightarrow L^{q}((0, \infty) ; u(t) d t)$ defined by $\widetilde{I_{\alpha}} f(t)=\frac{1}{\Gamma(\alpha)} \int_{\frac{t}{2}}^{t}(t-s)^{\alpha-1} f(s) d s$ is bounded. 
In particular, if $p=q, u(t)=t^{\eta-1}$ and $v(t)=t^{\nu-1}, t>0$. Then boundedness of $I^{\alpha}$ : $L^{p}((0, \infty) ; v(t) d t) \rightarrow L^{p}((0, \infty) ; u(t) d t)$ holds if and only if

$$
v<p, \quad \eta<(1-\alpha) p \quad \text { and } \quad \alpha+\frac{\eta}{p}=\frac{v}{p} .
$$

Next, we give Hardy-type inequality involving Riemann-Liouville integral for the case $p=q=1$ (cf. [18, Theorem 2.3]).

Lemma 2.7 Let $u, v$ be non-negative weight functions. If there exist $\beta \in[0,1], C>0$ such that

$$
\left(\int_{r}^{\infty}(t-r)^{(\alpha-1) \beta} u(t) d t\right)\left(\sup _{0<t<r} \operatorname{ess}(r-t)^{(\alpha-1)(1-\beta)}[v(t)]^{-1}\right) \leq C \quad \text { for all } r>0 .
$$

Then there exists constant $B>0$ such that $\int_{0}^{\infty}\left|\left(I^{\alpha} f\right)(t)\right| u(t) d t \leq B \int_{0}^{\infty}|f(t)| v(t) d t$.

Lemma 2.8 (Hardy-Young inequality [19, p. 245-246]) Let $0<b \leq \infty$ and $p \geq 1$. Then, for any measurable function $\Psi:(0, b) \rightarrow \mathbb{R}^{+}$and $\gamma>0$, the following inequality holds:

$$
\int_{0}^{b} t^{-\gamma p}\left(\int_{0}^{t} \Psi(s) \frac{d s}{s}\right)^{p} \frac{d t}{t} \leq \frac{1}{\gamma^{p}} \int_{0}^{b} s^{-\gamma p} \Psi(s)^{p} \frac{d s}{s} .
$$

Before constructing interpolation spaces in terms of $S_{\alpha}(t), T_{\alpha}(t)$, we draft a base by recalling the classical real interpolation spaces. For Banach spaces $X$ and $Y, L(X ; Y)$ stands for the set of all bounded linear operators from $X$ to $Y$. For $X=Y, L(X ; X)$ is indicated as $L(X)$.

\subsection{Real interpolation spaces}

In this subsection, we recall interpolation space for the case $Y \hookrightarrow X$, though for general theory of interpolation spaces, we direct [20] for the interested reader. Let $\left(X,\|\cdot\|_{X}\right)$, $\left(Y,\|\cdot\|_{Y}\right)$ be Banach spaces with $Y \hookrightarrow X$. A Banach space $E$ is called an intermediate space if $Y \hookrightarrow E \hookrightarrow X$. An intermediate space $E$ is said to be an interpolation space if for every $T \in L(X)$ such that $\left.T\right|_{Y} \in L(Y)$, we have $\left.T\right|_{E} \in L(E)$. A classical method known as $K$-method to produce a class of real interpolation spaces is recalled now.

For $x \in X, t>0$, set

$$
K(t, x ; X, Y):=\inf \left\{\left\|x_{1}\right\|_{X}+t\left\|x_{2}\right\|_{Y}: x=x_{1}+x_{2}, x_{1} \in X, x_{2} \in Y\right\}
$$

Let $0<\theta<1$. For $1 \leq p \leq \infty$, define the following spaces:

$$
(X, Y)_{\theta, p}=\left\{x \in X: t \mapsto \phi(t)=t^{-\theta} K(t, x ; X, Y) \in L_{*}^{p}(0, \infty)\right\},
$$

with the norm $\|x\|_{(X, Y)_{\theta, p}}=\|\phi\|_{L_{*}^{p}(0, \infty)}$.

Then $(X, Y)_{\theta, p}$ are known as real interpolation spaces. One important thing is to note that $K(t, x ; X, Y) \leq\|x\|_{X}$ as $Y \hookrightarrow X$. Hence, to prove $x \in(X, Y)_{\theta, p}$, it is adequate to prove that $t \mapsto \phi(t) \in L_{*}^{p}(0, a)$, and then $\|x\|_{(X, Y)_{\theta, p}}$ becomes equivalent to $\|x\|_{X}+\|\phi\|_{L_{*}^{p}(0, a)}$, for any fixed $a>0$. 
A well-known result is that interpolation space $(X, D(A))_{\theta, p}$ can also be represented in terms of semigroup as follows:

$$
(X, D(A))_{\theta, p}=\left\{x \in X: t \mapsto \psi(t)=t^{1-\theta}\|A T(t) x\|_{X} \in L_{*}^{p}(0,1)\right\}:=D_{A}(\theta, p),
$$

with the equivalent norm $\|x\|_{\theta, p}=\|x\|_{X}+\|\psi\|_{L_{*}^{p}(0,1)}$ (see, [1, p. 46]).

Definition 2.9 Let $\theta \in(0,1)$. An intermediate space $E$ is of $J_{\theta}$ class if $\exists c>0$ s.t.

$$
\|x\|_{E} \leq c\|x\|_{X}^{1-\theta}\|x\|_{Y}^{\theta} \quad \text { for all } x \in Y .
$$

In such a case, it is written as $E \in J_{\theta}(X, Y)$.

Definition 2.10 Let $\theta \in(0,1)$. An intermediate space $E$ is of $K_{\theta}$ class if $\exists k>0$ s.t.

$$
K(t, x ; X, Y) \leq k t^{\theta}\|x\|_{E} \quad \text { for all } x \in E, t>0 .
$$

In such case, it is written as $E \in K_{\theta}(X, Y)$.

One can observe that $(X, Y)_{\theta, p}$ belong to the class $J_{\theta}(X, Y) \cap K_{\theta}(X, Y)$ for every $p \in[1, \infty]$. For any $\theta \in(0,1)$, the domain of fractional power of the sectorial operator, $D\left(A^{\theta}\right)$ is not an interpolation space but belongs to the class $J_{\theta}(X, D(A)) \cap K_{\theta}(X, D(A))$. However, in a Hilbert space $X$, if $A$ is densely defined positive self-adjoint operator, then $D\left(A^{\theta}\right)$ is an interpolation space (cf. [1]).

Now, we recall the properties of the solution operators $S_{\alpha}(t), T_{\alpha}(t)$ in fractional calculus in order to find the motivation behind constructing new interpolation spaces.

Lemma 2.11 ([14]) Let $S_{\alpha}(t)$ be defined as (1.3). Then the following results hold.

(i) $S_{\alpha}(t) \in L(X)$ and $\exists B_{1}=B_{1}(\alpha)>0$ such that $\left\|S_{\alpha}(t)\right\|_{L(X)} \leq B_{1}, \forall t>0$.

(ii) For each $t>0, S_{\alpha}(t) \in L(X ; D(A))$, and $A S_{\alpha}(t) x=S_{\alpha}(t) A x$ if $x \in D(A)$. Moreover, $\exists B_{2}=B_{2}(\alpha)>0$ s.t. $\left\|A S_{\alpha}(t)\right\|_{L(X)} \leq B_{2} t^{-\alpha}, \forall t>0$.

(iii) $t \mapsto S_{\alpha}(t) \in C^{\infty}((0, \infty) ; L(X))$ and $\exists M_{n}=M_{n}(\alpha)>0$ such that $\left\|S_{\alpha}^{(n)}(t)\right\|_{L(X)} \leq M_{n} t^{-n}, \forall t>0$. Moreover, $S_{\alpha}(t)$ can be continued analytically to the sector $\sum_{\theta-\frac{\pi}{2}}$.

(iv) For each $x \in X, \lim _{t \rightarrow 0}\left\|S_{\alpha}(t) x-x\right\|_{X}=0$.

(v) $\frac{1}{\Gamma(\alpha)} \int_{0}^{t}(t-s)^{\alpha-1} S_{\alpha}(s) x d s \in D(A), \forall x \in D(A)$, and

$$
\frac{1}{\Gamma(\alpha)} \int_{0}^{t}(t-s)^{\alpha-1} A S_{\alpha}(s) x d s=S_{\alpha}(t) x-x
$$

Remark 2.12 Since $A$ is densely defined, closed operator, therefore (2.5) holds for all $x \in X$. Thus, the operator equation $\left(I^{\alpha} A S_{\alpha}\right)(t)=S_{\alpha}(t)-I$ is valid on $X$.

Lemma 2.13 ([14]) Let $T_{\alpha}(t)$ be defined as (1.4). Then the following results hold.

(i) $T_{\alpha}(t) \in L(X)$ and $\exists C_{1}=C_{1}(\alpha)>0$ such that $\left\|T_{\alpha}(t)\right\|_{L(X)} \leq C_{1} t^{\alpha-1}, \forall t>0$.

(ii) For each $t>0, T_{\alpha}(t) \in L(X ; D(A))$, and $A T_{\alpha}(t) x=T_{\alpha}(t) A x$ if $x \in D(A)$. Moreover, $\exists C_{2}=C_{2}(\alpha)>0$ such that $\left\|A T_{\alpha}(t)\right\|_{L(X)} \leq C_{2} t^{-1}, \forall t>0$. 
(iii) $t \mapsto T_{\alpha}(t) \in C^{\infty}((0, \infty) ; L(X))$, and $\exists K_{n}=K_{n}(\alpha)>0$ such that $\left\|T_{\alpha}^{(n)}(t)\right\|_{L(X)} \leq K_{n} t^{\alpha-n-1}, \forall t>0$. Moreover, $T_{\alpha}(t)$ can be continued analytically to the sector $\sum_{\theta-\frac{\pi}{2}}$.

(iv) $S_{\alpha}^{\prime}(t) x=A T_{\alpha}(t) x$ for all $x \in X, t>0$.

(v) $S_{\alpha}(t) x=\left(g_{1-\alpha} * T_{\alpha}\right)(t) x$ for all $x \in X, t>0$.

Note that $S_{\alpha}(t)$ is bounded near zero. Hence, by defining $S_{\alpha}(0)=I$, the identity operator on $X$ and taking note of the property given in Lemma 2.11(iv), we can say that the family of bounded linear operators $\left\{S_{\alpha}(t)\right\}_{t \geq 0}$ is continuous for $t \geq 0$ in the strong operator topology.

Lemma 2.14 Let $A: D(A) \subset X \rightarrow X$ be a densely defined sectorial operator and $S_{\alpha}(t), T_{\alpha}(t)$ be the fractional solutions operator defined as in (1.3), (1.4), respectively. Then the following identity holds:

$$
S_{\alpha}^{\prime \prime}(t) x=A T_{\alpha}^{\prime}(t) x \quad \text { for all } t>0, x \in X
$$

Proof Consider the following representations of $S_{\alpha}(t), T_{\alpha}(t)$ (see, [5, p. 213]):

$$
\begin{aligned}
& S_{\alpha}(t) x=\int_{0}^{\infty} M_{\alpha}(s) T\left(s t^{\alpha}\right) x d s, \\
& T_{\alpha}(t) x=t^{\alpha-1} \int_{0}^{\infty} \alpha s M_{\alpha}(s) T\left(s t^{\alpha}\right) x d s, \quad t>0, x \in X,
\end{aligned}
$$

where $M_{\alpha}$ is a probability density function having the property $\int_{0}^{\infty} s^{r} M_{\alpha}(s) d s=\frac{\Gamma(1+r)}{\Gamma(1+\alpha r)}, r>$ -1 .

Since $t \mapsto T_{\alpha}(t) \in C^{\infty}((0, \infty) ; L(X))$, using the Lebesgue dominated convergence theorem, we have the following integral representation of $T_{\alpha}^{\prime}(t)$ :

$$
T_{\alpha}^{\prime}(t) x=(\alpha-1) t^{-1} T_{\alpha}(t) x+t^{2(\alpha-1)} \int_{0}^{\infty} \alpha^{2} s^{2} M_{\alpha}(s) T^{\prime}\left(s t^{\alpha}\right) x d s
$$

Now, $\alpha^{2} s^{2} M_{\alpha}(s)\left\|A T^{\prime}\left(s t^{\alpha}\right)\right\|_{L(X)} \leq C t^{-2 \alpha} M_{\alpha}(s)$, which is integrable over $s \in(0, \infty)$. Hence, by [20, Proposition A.5.], we conclude that $T_{\alpha}^{\prime}(t) x \in D(A)$ for all $x \in X, t>0$.

Finally, using the identity $T^{(n)}(t)=A^{n} T(t), t>0, n \in \mathbb{N}$, analyticity of $t \mapsto S_{\alpha}(t)$ and the Lebesgue dominated convergence theorem, we get

$$
S_{\alpha}^{\prime \prime}(t) x=A T_{\alpha}^{\prime}(t) x \quad \text { for all } t>0, x \in X
$$

\section{Construction and characterization of the interpolation spaces in terms of $S_{\alpha}(t), T_{\alpha}(t)$}

In this section, we introduce two classes of interpolation spaces in terms of solution operators. We show that these interpolation spaces are identical with the classical real interpolation space.

Let $0<\theta<1,1 \leq p \leq \infty$. We define the following classes:

$$
{ }^{T_{\alpha}} D_{A}(\theta, p):=\left\{y \in X: t \mapsto \psi_{\alpha}(t)=t^{1-\alpha \theta}\left\|A T_{\alpha}(t) y\right\|_{X} \in L_{*}^{p}(0,1)\right\}
$$


equipped with the norm ${ }^{T_{\alpha}}\|y\|_{\theta, p}=\|y\|_{X}+\left\|\psi_{\alpha}\right\|_{L_{*}^{p}(0,1)}$.

$$
{ }^{S_{\alpha}} D_{A}(\theta, p):=\left\{y \in X: t \mapsto \phi_{\alpha}(t)=t^{\alpha(1-\theta)}\left\|A S_{\alpha}(t) y\right\|_{X} \in L_{*}^{p}(0,1)\right\}
$$

equipped with the norm ${ }^{S}\|y\|_{\theta, p}=\|y\|_{X}+\left\|\phi_{\alpha}\right\|_{L_{*}^{p}(0,1)}$.

Remark 3.1 We observe that, for any $0<a<b<\infty$, the two maps $t \mapsto \psi_{\alpha}(t)$ and $t \mapsto \phi_{\alpha}(t)$ belong to $L_{*}^{p}(a, b)$. Indeed, $\left\|\psi_{\alpha}\right\|_{L_{*}^{p}(a, b)} \leq C_{1}(\alpha, a, b, \theta, p)\|y\|_{X}$ and $\left\|\phi_{\alpha}\right\|_{L_{*}^{p}(a, b)} \leq$ $C_{2}(\alpha, a, b, \theta, p)\|y\|_{X}$ for some $C_{1}, C_{2}>0$. Therefore, one can replace the interval $(0,1)$ by any intervals of the form $(0, T]$ and check that the corresponding norms will also be equivalent to the norms mentioned in (3.1), (3.2), respectively.

From now onward, we will use $C$ as a positive constant appeared in any estimation instead of mentioning it precisely and the norm $\|\cdot\|$ in place of the norm $\|\cdot\|_{X}$ on $X$.

Theorem 3.2 Let $\theta \in(0,1), p \in[1, \infty]$. Then

$$
D_{A}(\theta, p)={ }^{T_{\alpha}} D_{A}(\theta, p)={ }^{S_{\alpha}} D_{A}(\theta, p),
$$

and the respective norms defined on these spaces are equivalent.

Proof Let $x \in D_{A}(\theta, p):=(X, D(A))_{\theta, p}$ with $x=x_{1}+x_{2}$, where $x_{1} \in X$ and $x_{2} \in D(A)$. By the properties in Lemma 2.13, $\left\|A T_{\alpha}(t) x\right\| \leq\left\|A T_{\alpha}(t) x_{1}\right\|+\left\|A T_{\alpha}(t) x_{2}\right\| \leq C\left(t^{-1}\left\|x_{1}\right\|+\right.$ $\left.t^{\alpha-1}\left\|A x_{2}\right\|\right)$.

This implies that

$$
\psi_{\alpha}(t)=t^{1-\alpha \theta}\left\|A T_{\alpha}(t) x\right\| \leq C t^{-\alpha \theta}\left(\left\|x_{1}\right\|+t^{\alpha}\left\|x_{2}\right\|_{D(A)}\right)=C t^{-\alpha \theta} K\left(t^{\alpha}, x ; X, D(A)\right) .
$$

By the change of variable $t \mapsto t^{\alpha}$, the R.H.S of (3.3) belongs to $L_{*}^{p}(0,1)$, which concludes that the map $t \mapsto \psi_{\alpha}(t) \in L_{*}^{p}(0,1)$, and ${ }^{T_{\alpha}}\|x\|_{\theta, p} \leq C\|x\|_{\theta, p}$.

Hence we proved that

$$
D_{A}(\theta, p) \hookrightarrow{ }^{T_{\alpha}} D_{A}(\theta, p) .
$$

Now, let $x \in{ }^{T_{\alpha}} D_{A}(\theta, p)$. By Lemma 2.13(v) and the closedness of $A$, we have

$$
\begin{aligned}
\left\|A S_{\alpha}(t) x\right\| & \leq \frac{1}{\Gamma(1-\alpha)} \int_{0}^{t}(t-s)^{-\alpha}\left\|A T_{\alpha}(s) x\right\| d s \\
& \leq \frac{1}{\Gamma(1-\alpha)} \int_{0}^{t}(t-s)^{-\alpha} s^{\alpha \theta-1} \psi_{\alpha}(s) d s, \quad \text { for } 0<t<1 .
\end{aligned}
$$

If $p=\infty$, then $\left\|A S_{\alpha}(t) x\right\| \leq \frac{1}{\Gamma(1-\alpha)} t^{\alpha(\theta-1)} B(1-\alpha, \alpha \theta)\left\|\psi_{\alpha}\right\|_{L^{\infty}(0,1)}$, where $B$ is the beta function defined as $B(m, n)=\int_{0}^{1}(1-s)^{m-1} s^{n-1} d s, m, n>0$.

Thus, the map $t \mapsto \phi_{\alpha}(t) \in L_{*}^{\infty}(0,1)$, and ${ }^{S_{\alpha}}\|x\|_{\theta, \infty} \leq C^{T_{\alpha}}\|x\|_{\theta, \infty}$.

For $1<p<\infty$, one can easily check the validity of the inequality (2.1) with the functions $u(t)=t^{\alpha(1-\theta) p-1}, v(t)=t^{(1-\alpha \theta) p-1}$. Thus, by Lemma 2.6 and noting Lemma 2.13(ii), (v) we 
get

$$
\begin{aligned}
& \int_{0}^{1} t^{\alpha(1-\theta) p}\left\|A S_{\alpha}(t) x\right\|^{p} \frac{d t}{t} \\
& \quad \leq \int_{0}^{\infty} t^{\alpha(1-\theta) p}\left\|I^{1-\alpha}\left(A T_{\alpha}\right)(t) x\right\|^{p} \frac{d t}{t} \\
& \quad \leq C \int_{0}^{\infty} t^{(1-\alpha \theta) p}\left\|A T_{\alpha}(t) x\right\|^{p} \frac{d t}{t} \\
& \quad \leq C\left(\int_{0}^{1} t^{(1-\alpha \theta) p}\left\|A T_{\alpha}(t) x\right\|^{p} \frac{d t}{t}+\int_{1}^{\infty} t^{(1-\alpha \theta) p-1} t^{-p}\|x\|^{p} d t\right) \\
& \quad \leq C\left(\int_{0}^{1} t^{(1-\alpha \theta) p}\left\|A T_{\alpha}(t) x\right\|^{p} \frac{d t}{t}+\|x\|^{p}\right) \\
& \quad=C\left(\left\|\psi_{\alpha}\right\|_{L_{*}^{p}(0,1)}^{p}+\|x\|^{p}\right),
\end{aligned}
$$

which resulted to ${ }^{S_{\alpha}}\|x\|_{\theta, p} \leq C^{T_{\alpha}}\|x\|_{\theta, p}$. Hence, ${ }^{T_{\alpha}} D_{A}(\theta, p) \hookrightarrow{ }^{S_{\alpha}} D_{A}(\theta, p)$.

For the case $p=1$, the inequality (2.2) holds for $u(t)=t^{\alpha(1-\theta)-1}, v(t)=t^{-\alpha \theta}$ and $\beta=1$. Again, by a similar procedure to the last case, we can obtain ${ }^{S_{\alpha}}\|x\|_{\theta, 1} \leq C^{T_{\alpha}}\|x\|_{\theta, 1}$. Hence, for all $1 \leq p \leq \infty$,

$$
{ }^{T_{\alpha}} D_{A}(\theta, p) \hookrightarrow{ }^{S_{\alpha}} D_{A}(\theta, p)
$$

Again, let $x \in{ }^{S_{\alpha}} D_{A}(\theta, p)$. Then, by Lemma 2.11(v), we can write

$$
x=-\left(S_{\alpha}(t) x-x\right)+S_{\alpha}(t) x=-\frac{1}{\Gamma(\alpha)} \int_{0}^{t}(t-s)^{\alpha-1} A S_{\alpha}(s) x d s+S_{\alpha}(t) x .
$$

Therefore,

$$
K\left(t^{\alpha}, x ; X, D(A)\right) \leq \frac{1}{\Gamma(\alpha)} \int_{0}^{t}(t-s)^{\alpha-1}\left\|A S_{\alpha}(s) x\right\| d s+t^{\alpha}\left\|S_{\alpha}(t) x\right\|_{D(A)}, \quad t>0 .
$$

First, consider $p=\infty$ :

$$
\begin{aligned}
K\left(t^{\alpha}, x ; X, D(A)\right) \leq & \frac{1}{\Gamma(\alpha)} \int_{0}^{t}(t-s)^{\alpha-1}\left\|A S_{\alpha}(s) x\right\| d s+t^{\alpha}\left\|S_{\alpha}(t) x\right\|_{D(A)} \\
\leq & \frac{1}{\Gamma(\alpha)} \int_{0}^{t}(t-s)^{\alpha-1} s^{\alpha(\theta-1)}\left\|\phi_{\alpha}\right\|_{L_{*}^{\infty}(0,1)} d s+C t^{\alpha}\|x\| \\
& +t^{\alpha(\theta-1)+\alpha}\left\|\phi_{\alpha}\right\|_{L_{*}^{\infty}(0,1)} \\
\leq & C t^{\alpha \theta}\left\|\phi_{\alpha}\right\|_{L_{*}^{\infty}(0,1)}+C t^{\alpha}\|x\|+t^{\alpha \theta}\left\|\phi_{\alpha}\right\|_{L_{*}^{\infty}(0,1)},
\end{aligned}
$$

implying $t^{-\alpha \theta} K\left(t^{\alpha}, x ; X, D(A)\right) \leq C t^{\alpha-\alpha \theta S_{\alpha}}\|x\|_{\theta, \infty}$.

Therefore, noting the change of variable $t \mapsto t^{\alpha}$, we obtain

$$
\|x\|_{D_{A}(\theta, \infty)} \leq C^{S_{\alpha}}\|x\|_{\theta, \infty} .
$$


Now, for the case $1 \leq p<\infty$, we first note that

$$
\int_{0}^{1} t^{-\alpha \theta p} K\left(t^{\alpha}, x ; X, D(A)\right)^{p} \frac{d t}{t} \leq \int_{0}^{1} t^{-\alpha \theta p}\left\|\left(I^{\alpha} A S_{\alpha}\right)(t) x\right\|^{p} \frac{d t}{t}+C\|x\|^{p}+\left\|\phi_{\alpha}\right\|_{L_{*}^{p}(0,1)}^{p} .
$$

Now, to prove $\|x\|_{D_{A}(\theta, p)} \leq C^{S_{\alpha}}\|x\|_{\theta, p}$, it suffices to show that

$$
\int_{0}^{1} t^{-\alpha \theta p}\left\|\left(I^{\alpha} A S_{\alpha}\right)(t) x\right\|^{p} \frac{d t}{t} \leq C \int_{0}^{1} t^{\alpha(1-\theta) p}\left\|A S_{\alpha}(t) x\right\|^{p} \frac{d t}{t} .
$$

This inequality immediately follows from Lemmas 2.6, 2.7 with $u(t)=t^{-\alpha \theta p-1}, v(t)=$ $t^{\alpha(1-\theta) p-1}, \beta=1$ and using the procedure same as in the proof of the second inclusion (inclusion number (3.5)). Hence for all $1 \leq p \leq \infty$,

$$
{ }^{S_{\alpha}} D_{A}(\theta, p) \hookrightarrow D_{A}(\theta, p) .
$$

Therefore, the inclusions (3.4), (3.5) and (3.7) validate the theorem.

Next we show the another formation of ${ }^{S_{\alpha}} D_{A}(\theta, p)$ in the next result.

Proposition 3.3 Let $0<\theta<1$ and $1 \leq p \leq \infty$. Then the following holds:

$$
{ }^{S_{\alpha}} D_{A}(\theta, p)=\left\{y \in X: t \mapsto \varphi_{\alpha}(t)=t^{-\alpha \theta}\left\|S_{\alpha}(t) y-y\right\| \in L_{*}^{p}(0,1)\right\},
$$

and the norm $y \mapsto{ }^{S_{\alpha}}\|y\|_{\theta, p}$ is equivalent to the norm $y \mapsto\|y\|+\left\|\varphi_{\alpha}\right\|_{L_{*}^{p}(0,1)}$.

Proof Let $x \in{ }^{S_{\alpha}} D_{A}(\theta, p)$, i.e., $x \in X$ such that $t \mapsto \phi_{\alpha}(t)=t^{\alpha(1-\theta)}\left\|A S_{\alpha}(t) x\right\| \in L_{*}^{p}(0,1)$.

For $p=\infty$. By Lemma 2.11(v), we get

$$
\begin{aligned}
\left\|S_{\alpha}(t) x-x\right\| & =\left\|\left(I^{\alpha} A S_{\alpha}\right)(t) x\right\| \\
& \leq \frac{1}{\Gamma(\alpha)} \int_{0}^{t}(t-s)^{\alpha-1} s^{\alpha(\theta-1)} \phi_{\alpha}(s) d s \\
& \leq C t^{\alpha \theta}\left\|\phi_{\alpha}\right\|_{L_{*}^{\infty}(0,1) \quad \text { for all } t \in(0,1) .}
\end{aligned}
$$

Then it is obvious that $\varphi_{\alpha} \in L_{*}^{\infty}(0,1)$ and $\left\|\varphi_{\alpha}\right\|_{L_{*}^{\infty}(0,1)} \leq C\left\|\phi_{\alpha}\right\|_{L_{*}^{\infty}(0,1)}$.

For $1 \leq p<\infty$, it is adequate to prove that $\exists C>0$ such that

$$
\int_{0}^{1} t^{-\theta \alpha p}\left\|\left(I^{\alpha} A S_{\alpha}\right)(t) x\right\|^{p} \frac{d t}{t} \leq C \int_{0}^{1} t^{\alpha(1-\theta) p}\left\|A S_{\alpha}(t) x\right\|^{p} \frac{d t}{t} .
$$

This inequality appeared in the proof of the third inclusion of Theorem 3.2 and has been proved by using Lemmas 2.6, 2.7 (see the inequality (3.6)).

Conversely, let $y \in X$ such that $t \mapsto \varphi_{\alpha}(t)=t^{-\alpha \theta}\left\|S_{\alpha}(t) y-y\right\| \in L_{*}^{p}(0,1)$.

Now one can check the validity of the following identity for all $t>0$ :

$$
\begin{aligned}
A S_{\alpha}(t) y= & A S_{\alpha}(t) \frac{\alpha}{t^{\alpha}} \int_{0}^{t}(t-s)^{\alpha-1}\left(y-S_{\alpha}(s) y\right) d s \\
& +A S_{\alpha}(t) \frac{\Gamma(\alpha+1)}{t^{\alpha}} \frac{1}{\Gamma(\alpha)} \int_{0}^{t}(t-s)^{\alpha-1} S_{\alpha}(s) y d s .
\end{aligned}
$$


By using Lemma 2.11(v), we get

$$
A S_{\alpha}(t) y=A S_{\alpha}(t) \frac{\alpha}{t^{\alpha}} \int_{0}^{t}(t-s)^{\alpha-1}\left(y-S_{\alpha}(s) y\right) d s+S_{\alpha}(t) \frac{\Gamma(\alpha+1)}{t^{\alpha}}\left(S_{\alpha}(t) y-y\right) .
$$

For the case $p=\infty$, we employ the properties in Lemma 2.11 and get

$$
\begin{aligned}
\left\|A S_{\alpha}(t) y\right\| & \leq C t^{-2 \alpha} \int_{0}^{t}(t-s)^{\alpha-1} s^{\alpha \theta} \varphi_{\alpha}(s) d s+C t^{\alpha(\theta-1)} \varphi_{\alpha}(t) \\
& \leq 2 C t^{\alpha(\theta-1)}\left\|\varphi_{\alpha}\right\|_{L_{*}^{\infty}(0,1)}
\end{aligned}
$$

So $t \mapsto \phi_{\alpha}(t)=t^{\alpha(1-\theta)}\left\|A S_{\alpha}(t) y\right\|$ belongs to $L_{*}^{\infty}(0,1)$, and $\left\|\phi_{\alpha}\right\|_{L_{*}^{\infty}(0,1)} \leq C\left\|\varphi_{\alpha}\right\|_{L_{*}^{\infty}(0,1)}$.

For the case $1 \leq p<\infty$, first note that

$$
t^{\alpha(1-\theta)}\left\|A S_{\alpha}(t) y\right\| \leq C\left(t^{-\alpha(1+\theta)}\left\|\left(I^{\alpha}\left(y-S_{\alpha}(\cdot) y\right)\right)(t)\right\|+\varphi_{\alpha}(t)\right)
$$

Hence, to prove $\left\|\phi_{\alpha}\right\|_{L_{*}^{p}(0,1)} \leq C\left\|\varphi_{\alpha}\right\|_{L_{*}^{p}(0,1)}$, it is enough to prove that

$$
\int_{0}^{1} t^{-\alpha(1+\theta) p}\left\|\left(I^{\alpha}\left(y-S_{\alpha}(\cdot) y\right)\right)(t)\right\|^{p} \frac{d t}{t} \leq C \int_{0}^{1} t^{-\alpha \theta p}\left\|y-S_{\alpha}(t) y\right\|^{p} \frac{d t}{t}
$$

The above inequality is validated by the Lemmas $2.6,2.7$ for $u(t)=t^{-\alpha(1+\theta) p-1}, v(t)=t^{-\alpha \theta p-1}$ and $\beta=1$. Hence, the proposition is proved.

\section{Strict regularity of mild solutions}

This section is devoted to establishing strict Hölder regularity (i.e., the $p=\infty$ case) results using the interpolation space constructed in the last section. Before that we shall present the necessary and sufficient condition for a mild solution to be a strict solution of the problem (1.2) in the case $0<\alpha<1$. For the case $1<\alpha<2$, we refer to [21].

Proposition 4.1 Let $\mathfrak{F} \in C([0, T] ; X), v_{0} \in D(A)$, and $v$ be a mild solution of $(1.2)$ on $[0, T]$. Let us consider

$$
w(t)=\int_{0}^{t} T_{\alpha}(t-s) \mathfrak{F}(s) d s, \quad t>0
$$

Then the following are equivalent:

(i) $v$ is strict solution of (1.2).

(ii) $w(t) \in D(A), \forall t \in[0, T]$, and $w \in C([0, T] ; D(A))$.

Proof Let $v$ be a strict solution. Then $\left.v \in C([0, T] ; D(A)), I^{1-\alpha}\left(v(\cdot)-v_{0}\right)\right) \in C^{1}([0, T] ; X)$ and $v$ satisfies Eq. (1.2), and explicitly $v(t)=S_{\alpha}(t) v_{0}+w(t)$. Since by Lemma $2.11 S_{\alpha}(t) v_{0} \in$ $D(A), \forall t \in[0, T]$, we have $w(t) \in D(A)$ and

$$
A w(t)=A v(t)-A S_{\alpha}(t) v_{0}=A v(t)-S_{\alpha}(t) A v_{0} .
$$

Hence, it follows that $w \in C([0, T] ; D(A))$.

Now, we assume that $w(t) \in D(A), \forall t \in[0, T]$, and $w \in C([0, T] ; D(A))$. 
Then we prove that $\left.v \in C([0, T] ; D(A)), I^{1-\alpha}\left(v(\cdot)-v_{0}\right)\right) \in C^{1}([0, T] ; X)$ and $v$ satisfies Eq. (1.2).

Since $v$ is mild solution of $(1.2), v(t)=S_{\alpha}(t) v_{0}+w(t)$. Obviously $v \in C([0, T] ; D(A))$.

Now

$$
\begin{aligned}
\left(g_{1-\alpha} *\left(v(\cdot)-v_{0}\right)\right)(t) & =\left(g_{1-\alpha} *\left(S_{\alpha}(\cdot) v_{0}-v_{0}\right)\right)(t)+\left(g_{1-\alpha} * T_{\alpha} * \mathfrak{F}\right)(t) \\
& =\left(g_{1-\alpha} * g_{\alpha} * A S_{\alpha}(\cdot) v_{0}\right)(t)+\left(S_{\alpha} * \mathfrak{F}\right)(t) \\
& =\left(g_{1} * A S_{\alpha}(\cdot) v_{0}\right)(t)+\left(S_{\alpha} * \mathfrak{F}\right)(t) .
\end{aligned}
$$

Also, by Remark 2.12 we have

$$
\begin{aligned}
\left(S_{\alpha} * \mathfrak{F}\right)(t) & =A\left(g_{\alpha} * S_{\alpha} * \mathfrak{F}\right)(t)+\left(g_{1} * \mathfrak{F}\right)(t) \\
& =A\left(g_{\alpha} * g_{1-\alpha} * T_{\alpha} * \mathfrak{F}\right)(t)+\left(g_{1} * \mathfrak{F}\right)(t) \\
& =A\left(g_{1} * T_{\alpha} * \mathfrak{F}\right)(t)+\left(g_{1} * \mathfrak{F}\right)(t) \\
& =\left(g_{1} * A w\right)(t)+\left(g_{1} * \mathfrak{F}\right)(t) .
\end{aligned}
$$

Since $A w \in C([0, T] ; X)$ and $\mathfrak{F} \in C([0, T] ; X)$, it follows that $t \mapsto\left(S_{\alpha} * \mathfrak{F}\right)(t)$ belongs to $C^{1}([0, T] ; X)$. Also $A S_{\alpha}(\cdot) v_{0} \in C([0, T] ; X)$ as $v_{0} \in D(A)$. So, $t \mapsto\left(g_{1} * A S_{\alpha}(\cdot) v_{0}\right)(t)$ belongs to $C^{1}([0, T] ; X)$. Consequently, $I^{1-\alpha}\left(v(\cdot)-v_{0}\right)=\left(g_{1-\alpha} *\left(v(\cdot)-v_{0}\right)\right) \in C^{1}([0, T] ; X)$. Now using (4.1), (4.2),

$$
\begin{aligned}
{ }^{c} D_{t}^{\alpha} v(t) & =\frac{d}{d t}\left(g_{1-\alpha} *\left(v(\cdot)-v_{0}\right)\right)(t) \\
& =A S_{\alpha}(t) v_{0}+\frac{d}{d t}\left(S_{\alpha} * \mathfrak{F}\right)(t) \\
& =A S_{\alpha}(t) v_{0}+A w(t)+\mathfrak{F}(t) \\
& =A v(t)+\mathfrak{F}(t) .
\end{aligned}
$$

Proposition 4.2 Consider the integral defined as

$$
w(t)=\int_{0}^{t} T_{\alpha}(t-s) \mathfrak{F}(s) d s, \quad \mathfrak{F} \in C([0, T] ; X) .
$$

Then $w \in C^{\alpha}([0, T] ; X)$.

Proof By Lemma 2.13, $\|w(t)\| \leq C\|\mathfrak{F}\|_{C([0, T] ; X)} \int_{0}^{t}(t-s)^{\alpha-1} d s \leq C\|\mathfrak{F}\|_{C([0, T] ; X)} t^{\alpha}$, for all $t \in$ $[0, T]$, with $w(0)=0$.

For $0 \leq s<t \leq T$,

$$
\begin{aligned}
\|w(t)-w(s)\| & \leq \int_{0}^{s}\left\|\left[T_{\alpha}(t-\tau)-T_{\alpha}(s-\tau)\right] \mathfrak{F}(\tau)\right\| d \tau+\int_{s}^{t}\left\|T_{\alpha}(t-\tau) \mathfrak{F}(\tau)\right\| d \tau \\
& \leq \int_{0}^{s} \int_{s-\tau}^{t-\tau}\left\|T_{\alpha}^{\prime}(\sigma) \mathfrak{F}(\tau)\right\| d \sigma d \tau+C\|\mathfrak{F}\|_{C([0, T] ; X)} \int_{s}^{t}(t-\tau)^{\alpha-1} d \tau \\
& \leq C\|\mathfrak{F}\|_{C([0, T] ; X)}\left(\int_{0}^{s} \int_{s-\tau}^{t-\tau} \sigma^{\alpha-2} d \sigma d \tau+\int_{s}^{t}(t-\tau)^{\alpha-1} d \tau\right)
\end{aligned}
$$




$$
\leq C\|\mathfrak{F}\|_{C([0, T] ; X)}(t-s)^{\alpha} .
$$

Hence, $w \in C^{\alpha}([0, T] ; X)$ and $[w]_{\alpha} \leq C\|\mathfrak{F}\|_{C([0, T] ; X)}$.

Theorem 4.3 Let $\mathfrak{F} \in C^{\theta}([0, T] ; X)$, where $\theta \in(0,1)$, and $v$ be the mild solution of (1.2). Also, we assume that $v_{0} \in D(A)$ such that $A v_{0}+\mathfrak{F}(0) \in{ }^{T_{\alpha}} D_{A}(\theta, \infty)$. Then $v$ is strict solution on $[0, T]$. Indeed, $v \in C^{\alpha \theta}([0, T] ; D(A))$ and consequently ${ }^{c} D_{t}^{\alpha} v \in C^{\alpha \theta}([0, T] ; X)$. Moreover, there exists $C>0$ such that

$$
\begin{aligned}
& \left\|{ }^{c} D_{t}^{\alpha} v\right\|_{C^{\alpha \theta}([0, T] ; X)}+\|A v\|_{C^{\alpha \theta}([0, T] ; X)} \\
& \quad \leq C\left(\|\mathfrak{F}\|_{C^{\theta}([0, T] ; X)}+\left\|v_{0}\right\|_{D(A)}+{ }^{T}\left\|A v_{0}+\mathfrak{F}(0)\right\|_{\theta, \infty}\right) .
\end{aligned}
$$

Proof We can write

$$
\begin{aligned}
v(t) & =S_{\alpha}(t) v_{0}+\int_{0}^{t} T_{\alpha}(t-s)(\mathfrak{F}(s)-\mathfrak{F}(t)) d s+\int_{0}^{t} T_{\alpha}(t-s) \mathfrak{F}(t) d s \\
& =S_{\alpha}(t) v_{0}+w_{1}(t)+\left(g_{1} * T_{\alpha}\right)(t) \mathfrak{F}(t) \\
& =S_{\alpha}(t) v_{0}+w_{1}(t)+\left(g_{\alpha} * S_{\alpha}\right)(t) \mathfrak{F}(t) \\
& =w_{1}(t)+w_{2}(t),
\end{aligned}
$$

where $w_{1}(t)=\int_{0}^{t} T_{\alpha}(t-s)(\mathfrak{F}(s)-\mathfrak{F}(t)) d s, w_{2}(t)=S_{\alpha}(t) v_{0}+\left(g_{\alpha} * S_{\alpha}\right)(t) \mathfrak{F}(t)$.

Now, $\left\|A T_{\alpha}(t-s)(\mathfrak{F}(s)-\mathfrak{F}(t))\right\| \leq C[\mathfrak{F}]_{\theta}(t-s)^{\theta-1}$, which is integrable on $(0, t)$. By the closedness of $A$, we have

$$
\left\|A w_{1}(t)\right\| \leq C[\mathfrak{F}]_{\theta} t^{\theta} \quad \text { for all } t \in[0, T] .
$$

Hence, $w_{1}(t) \in D(A), \forall t \in[0, T]$.

For $0 \leq s<t \leq T$, we have

$$
\begin{aligned}
\left\|A w_{1}(t)-A w_{1}(s)\right\| & \int_{0}^{s}\left\|\left(A T_{\alpha}(t-\sigma)-A T_{\alpha}(s-\sigma)\right)(\mathfrak{F}(\sigma)-\mathfrak{F}(s))\right\| d \sigma \\
& +\left\|\int_{0}^{s} A T_{\alpha}(t-\sigma)(\mathfrak{F}(s)-\mathfrak{F}(t)) d \sigma\right\|+\int_{s}^{t}\left\|A T_{\alpha}(t-\sigma)(\mathfrak{F}(\sigma)-\mathfrak{F}(t))\right\| d \sigma \\
\leq & \int_{0}^{s} \int_{s-\sigma}^{t-\sigma}\left\|A T_{\alpha}^{\prime}(\tau)\right\|_{L(X)}\|\mathfrak{F}(\sigma)-\mathfrak{F}(s)\| d \tau d \sigma \\
& +\left\|\int_{t-s}^{t} A T_{\alpha}(\tau)(\mathfrak{F}(s)-\mathfrak{F}(t)) d \tau\right\|+C[\mathfrak{F}]_{\theta} \int_{s}^{t}(t-\sigma)^{\theta-1} d \sigma \\
\leq & {[\mathfrak{F}]_{\theta} \int_{0}^{s}(s-\sigma)^{\theta} \int_{s-\sigma}^{t-\sigma}\left\|S_{\alpha}^{\prime \prime}(\tau)\right\|_{L(X)} d \tau d \sigma+\left\|\int_{t-s}^{t} S_{\alpha}^{\prime}(\tau)(\mathfrak{F}(s)-\mathfrak{F}(t)) d \tau\right\| } \\
& +C[\mathfrak{F}]_{\theta}(t-s)^{\theta} \\
\leq & C[\mathfrak{F}]_{\theta} \int_{0}^{s} \int_{s-\sigma}^{t-\sigma} \tau^{\theta-2} d \tau d \sigma+\left\|\left(S_{\alpha}(t)-S_{\alpha}(t-s)\right)(\mathfrak{F}(s)-\mathfrak{F}(t))\right\|
\end{aligned}
$$




$$
\begin{aligned}
& +C[\mathfrak{F}]_{\theta}(t-s)^{\theta} \\
\leq & C[\mathfrak{F}]_{\theta}(t-s)^{\theta}+2 B_{1}[\mathfrak{F}]_{\theta}(t-s)^{\theta}+C[\mathfrak{F}]_{\theta}(t-s)^{\theta} \\
\leq & C[\mathfrak{F}]_{\theta}(t-s)^{\theta} \leq C T^{\theta(1-\alpha)}[\mathfrak{F}]_{\theta}(t-s)^{\alpha \theta} .
\end{aligned}
$$

By Remark 2.12, we also note that

$$
A w_{2}(t)=S_{\alpha}(t) A v_{0}+\left(g_{\alpha} * A S_{\alpha}\right)(t) \mathfrak{F}(t)=S_{\alpha}(t)\left(A v_{0}+\mathfrak{F}(0)\right)+S_{\alpha}(t)(\mathfrak{F}(t)-\mathfrak{F}(0))-\mathfrak{F}(t) .
$$

For $0 \leq s<t \leq T$,

$$
\begin{aligned}
\left\|\left(S_{\alpha}(t)-S_{\alpha}(s)\right)\left(A v_{0}+\mathfrak{F}(0)\right)\right\| & \leq \int_{s}^{t}\left\|S_{\alpha}^{\prime}(\tau)\left(A v_{0}+\mathfrak{F}(0)\right)\right\| d \tau \\
& \leq \int_{s}^{t}\left\|A T_{\alpha}(\tau)\left(A v_{0}+\mathfrak{F}(0)\right)\right\| d \tau \\
& \leq \int_{s}^{t} \tau^{\alpha \theta-1 T_{\alpha}}\left\|A v_{0}+\mathfrak{F}(0)\right\|_{\theta, \infty} d \tau \\
& \leq C^{T_{\alpha}}\left\|A v_{0}+\mathfrak{F}(0)\right\|_{\theta, \infty}(t-s)^{\alpha \theta}
\end{aligned}
$$

and

$$
\begin{aligned}
& \left\|S_{\alpha}(t)(\mathfrak{F}(t)-\mathfrak{F}(0))-S_{\alpha}(s)(\mathfrak{F}(s)-\mathfrak{F}(0))\right\| \\
& \quad \leq\left\|\left(S_{\alpha}(t)-S_{\alpha}(s)\right)(\mathfrak{F}(s)-\mathfrak{F}(0))\right\|+\left\|S_{\alpha}(t)(\mathfrak{F}(t)-\mathfrak{F}(s))\right\| \\
& \quad \leq[\mathfrak{F}]_{\theta} \int_{s}^{t}\left\|S_{\alpha}^{\prime}(\tau)\right\|_{L(X)} s^{\theta} d \tau+C[\mathfrak{F}]_{\theta}(t-s)^{\theta} \\
& \quad \leq C[\mathfrak{F}]_{\theta} \int_{s}^{t} \tau^{\theta-1} d \tau+C[\mathfrak{F}]_{\theta}(t-s)^{\theta} \\
& \quad \leq C[\mathfrak{F}]_{\theta}(t-s)^{\theta} \leq C[\mathfrak{F}]_{\theta} T^{\theta(1-\alpha)}(t-s)^{\alpha \theta} .
\end{aligned}
$$

Hence, by (4.5), it is clear that $A w_{2} \in C^{\alpha \theta}([0, T] ; X)$ with $\left[A w_{2}\right]_{\alpha \theta} \leq C\left([\mathfrak{F}]_{\theta}+{ }^{T_{\alpha}} \| A v_{0}+\right.$ $\left.\mathfrak{F}(0) \|_{\theta, \infty}\right)$. Also, from (4.4), $A w_{1} \in C^{\alpha \theta}([0, T] ; X)$ with $\left[A w_{1}\right]_{\alpha \theta} \leq C[\mathfrak{F}]_{\theta}$.

Consequently, $A v \in C^{\alpha \theta}([0, T] ; X)$.

By Proposition 4.2, it follows that $v \in C^{\alpha \theta}([0, T] ; D(A))$ and the estimation (4.3) follows easily.

We say $\mathcal{B}$, set of all $X$-valued function on [0,T], satisfies maximal regularity property for the problem (1.2) if for every $\mathfrak{F} \in \mathcal{B}$, the solution $v$ of (1.2) is such that both ${ }^{c} D_{t}^{\alpha} v$ and $A v$ belong to $\mathcal{B}$. Clearly, if $0<\theta<\alpha$, then by Theorem $4.3, \mathcal{B}=C^{\theta}([0, T] ; X)$ enjoys the maximal regularity property for the problem (1.2) which is stated now as a corollary.

Corollary 4.4 Let $\mathfrak{F} \in C^{\theta}([0, T] ; X), \theta \in(0, \alpha)$. Assume that $v_{0} \in D(A)$ such that $A v_{0}+$ $\mathfrak{F}(0) \in{ }^{T_{\alpha}} D_{A}\left(\frac{\theta}{\alpha}, \infty\right)$. Then a mild solution, say $v$, of $(1.2)$ is a strict solution. Indeed, $v \in$ $C^{\theta}([0, T] ; D(A))$ and consequently ${ }^{c} D_{t}^{\alpha} v \in C^{\theta}([0, T] ; X)$. Moreover, there exists $C>0$ such 
that

$$
\begin{aligned}
& \left\|{ }^{c} D_{t}^{\alpha} \nu\right\|_{C^{\theta}([0, T] ; X)}+\|A v\|_{C^{\theta}([0, T] ; X)} \\
& \quad \leq C\left(\|\mathfrak{F}\|_{C^{\theta}([0, T] ; X)}+\left\|v_{0}\right\|_{D(A)}+{ }^{T}\left\|A v_{0}+\mathfrak{F}(0)\right\|_{\frac{\theta}{\alpha}, \infty}\right) .
\end{aligned}
$$

Corollary 4.5 Let $\mathfrak{F} \in C^{\theta}([0, T] ; X), \theta \in(0,1)$. Assume that $v_{0} \in D(A)$ such that $A v_{0}+$ $\mathfrak{F}(0)=0$. Then a mild solution, say $v$, of $(1.2)$ is a strict solution. Indeed, $v \in C^{\theta}([0, T] ; D(A))$ and consequently ${ }^{c} D_{t}^{\alpha} v \in C^{\theta}([0, T] ; X)$. Moreover, there exists $C>0$ such that

$$
\left\|{ }^{c} D_{t}^{\alpha} v\right\|_{C^{\theta}([0, T] ; X)}+\|A v\|_{C^{\theta}([0, T] ; X)} \leq C\left(\|\mathfrak{F}\|_{C^{\theta}([0, T] ; X)}+\left\|v_{0}\right\|_{D(A)}\right) .
$$

Remark 4.6 Now, one may question the establishment of the strict regularity of mild solution of (1.2) in the interpolation space ${ }^{T_{\alpha}} D_{A}(\theta, \infty)$ using the method presented in this paper. This is possible when $T_{\alpha}(t) y \in D\left(A^{2}\right), \forall t>0, y \in X$. However, till now it is an open problem to find whether $T_{\alpha}(t) y \in D\left(A^{2}\right), \forall t>0, y \in X$.

In [16], we studied that the assumptions $\nu_{0} \in D(A)$ and $\mathfrak{F} \in C^{\theta}([0, T] ; X)$ with $\mathfrak{F}(0) \neq 0$ do not guarantee that the mild solution is strict. But, in the next theorem, we shall see that the assumption $\mathfrak{F} \in C^{\theta}\left([0, T] ;^{T_{\alpha}} D_{A}(\theta, \infty)\right)$ ensures the strict Hölder regularity of a mild solution of (1.2). We shall use the following constant:

$$
M=\sup _{0<t \leq T+1} t^{1-\alpha \theta}\left\|A T_{\alpha}(t)\right\|_{L\left(T_{\alpha} D_{A}(\theta, \infty) ; X\right)} \cdot
$$

Theorem 4.7 Let $\mathfrak{F} \in C^{\theta}\left([0, T] ;{ }^{T_{\alpha}} D_{A}(\theta, \infty)\right), \theta \in(0,1)$, and $v_{0} \in D(A)$. Then a mild solution, say $v$, of (1.2) is a strict solution. Additionally, if we assume that $A v_{0} \in{ }^{T_{\alpha}} D_{A}(\theta, \infty)$, then $v \in C^{\alpha \theta}([0, T] ; D(A))$ and consequently ${ }^{c} D_{t}^{\alpha} v \in C^{\alpha \theta}([0, T] ; X)$. Moreover, there exists $C>0$ such that

$$
\begin{aligned}
& \left\|{ }^{c} D_{t}^{\alpha} \nu\right\|_{C^{\alpha \theta}([0, T] ; X)}+\|A v\|_{C^{\alpha \theta}([0, T] ; X)} \\
& \quad \leq C\left(\|\mathfrak{F}\|_{C^{\theta}\left([0, T] ; T^{\prime} D_{A}(\theta, \infty)\right)}+\left\|v_{0}\right\|_{D(A)}+{ }^{T_{\alpha}}\left\|A v_{0}\right\|_{\theta, \infty}\right) .
\end{aligned}
$$

Proof Let $w(t)=\int_{0}^{t} T_{\alpha}(t-s) \mathfrak{F}(s) d s, t>0$. Now, for $s \in(0, t)$ we have,

$$
\begin{aligned}
\left\|A T_{\alpha}(t-s) \mathfrak{F}(s)\right\| & \leq\left\|A T_{\alpha}(t-s)\right\|_{L\left(T_{\alpha} D_{A}(\theta, \infty) ; X\right)}\|\mathfrak{F}(s)\|_{T_{\alpha} D_{A}(\theta, \infty)} \\
& \leq M(t-s)^{\alpha \theta-1}\|\mathfrak{F}\|_{C^{\theta}\left([0, T] ; T^{T} D_{A}(\theta, \infty)\right)}
\end{aligned}
$$

which is integrable on $(0, t)$. Using the closedness of $A$, we get

$$
\|A w(t)\| \leq \frac{M}{\alpha \theta} t^{\alpha \theta}\|\mathfrak{F}\|_{C^{\theta}\left([0, T] ; T^{T_{\alpha}} D_{A}(\theta, \infty)\right)}<\infty, \forall t \in[0, T] \quad \text { with } A w(0)=0 .
$$

Therefore, $w(t) \in D(A)$ for all $t \in[0, T]$. Now let $0<z<1$ be such that $t, t+z \in[0, T]$. Then

$$
\begin{aligned}
& \|A w(t+z)-A w(t)\| \\
& \quad=\left\|\int_{0}^{t+z} A T_{\alpha}(t+z-\sigma) \mathfrak{F}(\sigma) d \sigma-\int_{0}^{t} A T_{\alpha}(t-\sigma) \mathfrak{F}(\sigma) d \sigma\right\|
\end{aligned}
$$




$$
\begin{aligned}
& =\left\|\int_{-z}^{t} A T_{\alpha}(t-\sigma) \mathfrak{F}(\sigma+z) d \sigma-\int_{0}^{t} A T_{\alpha}(t-\sigma) \mathfrak{F}(\sigma) d \sigma\right\| \\
& \leq \int_{-z}^{0}\left\|A T_{\alpha}(t-\sigma) \mathfrak{F}(\sigma+z)\right\| d \sigma+\int_{0}^{t}\left\|A T_{\alpha}(t-\sigma)(\mathfrak{F}(\sigma+z)-\mathfrak{F}(\sigma))\right\| d \sigma \\
& \leq M\|\mathfrak{F}\|_{C^{\theta}\left([0, T] ; ;^{T} D_{A}(\theta, \infty)\right)}\left(\int_{-z}^{0}(t-\sigma)^{\alpha \theta-1} d \sigma+z^{\theta} \int_{0}^{t}(t-\sigma)^{\alpha \theta-1} d \sigma\right) \\
& \leq C\|\mathfrak{F}\|_{C^{\theta}\left([0, T] ;{ }^{T} D_{A}(\theta, \infty)\right)} z^{\alpha \theta} .
\end{aligned}
$$

Therefore, $A w \in C^{\alpha \theta}([0, T] ; X)$ and by Proposition 4.2, $w \in C^{\alpha \theta}([0, T] ; D(A))$. Hence by Proposition 4.1, it is concluded that $v$ is a strict solution of (1.2).

Now, if $A v_{0} \in{ }^{T_{\alpha}} D_{A}(\theta, \infty)$, then by following the procedure to obtain (4.5) in Theorem 4.3, we can prove that $t \mapsto A S_{\alpha}(t) v_{0} \in C^{\alpha \theta}([0, T] ; X)$ with $\left[A S_{\alpha}(\cdot) v_{0}\right]_{\alpha \theta} \leq{ }^{T_{\alpha}}\left\|v_{0}\right\|_{\theta, \infty}$ and the estimation (4.9) follows easily.

Example 4.8 Consider the following time-fractional diffusion equation:

$$
\begin{aligned}
& { }^{c} D_{t}^{\alpha} u(t, x)=\Delta u(t, x)+f(t, x), \quad t \in(0, T], x \in \mathbb{R}^{n}, \alpha \in(0,1), \\
& u(0, x)=u_{0}(x), \quad x \in \mathbb{R}^{n} .
\end{aligned}
$$

Consider the operator $A: D(A) \rightarrow X=C_{b}\left(\mathbb{R}^{n}\right)$ defined by

$$
\left\{\begin{array}{l}
D(A)=\left\{u \in \bigcap_{1 \leq p<\infty} W_{\text {loc }}^{2, p}\left(\mathbb{R}^{n}\right) \cap C_{b}\left(\mathbb{R}^{n}\right): \Delta u \in C_{b}\left(\mathbb{R}^{n}\right)\right\} \\
A u=\Delta u,
\end{array}\right.
$$

where $C_{b}\left(\mathbb{R}^{n}\right)$ is the space of all bounded continuous functions endowed with the usual supremum norm. The operator $A$ generates an analytic semigroup $e^{t A}$ and $\overline{D(A)}=$ $\operatorname{BUC}\left(\mathbb{R}^{n}\right):=X_{0}$, the space of all bounded uniformly continuous functions (cf. [1, p. 81]). Now, the part of $A$ in $X_{0}$ is defined by

$$
D\left(A_{0}\right)=\left\{u \in D(A): A u \in X_{0}\right\}, \quad A_{0} u:=A u, u \in D\left(A_{0}\right) .
$$

Then $A_{0}: D\left(A_{0}\right) \rightarrow X_{0}$ is densely defined sectorial operator which generates the bounded analytic semigroup $e^{t A_{0}}$. In fact, $e^{t A_{0}}=e^{t A}$ (cf. [1, Remark 2.1.5]). Now, using the setting $u(t, x)=u(t)(x)$ and $f(t, x)=f(t)(x)$, the problem (4.10) can be written as the following abstract Cauchy problem in the Banach space $X_{0}$ :

$$
\left\{\begin{array}{l}
{ }^{c} D_{t}^{\alpha} u(t)=A_{0} u(t)+f(t), \quad t>0 \\
u(0)=u_{0}
\end{array}\right.
$$

We denote $C_{b}^{\theta}\left(\mathbb{R}^{n}\right)$ as the space of all bounded and Hölder continuous functions endowed with the usual Hölder norm. We also define $C_{b}^{2+\theta}\left(\mathbb{R}^{n}\right)=\left\{f: D^{\beta} f\right.$ exists, $D^{\beta} f \in$ $C_{b}\left(\mathbb{R}^{n}\right) \forall$ multi-index $\beta$ with $|\beta| \leq 2$, and $D^{\beta} f \in C_{b}^{\theta}\left(\mathbb{R}^{n}\right)$ for $|\beta|=2$ \}.

By Theorem 3.2, [1, p. 45] and [1, Theorem 3.1.12], we know that

$$
{ }^{T_{\alpha}} D_{A}(\theta, \infty)={ }^{S_{\alpha}} D_{A}(\theta, \infty)=D_{A}(\theta, \infty)=D_{A_{0}}(\theta, \infty)=C_{b}^{2 \theta}\left(\mathbb{R}^{n}\right) .
$$

Therefore, $u_{0} \in C_{b}^{2+2 \theta}\left(\mathbb{R}^{n}\right)$ implies that $u_{0} \in D\left(A_{0}\right)$ and $A_{0} u_{0} \in{ }^{T_{\alpha}} D_{A}(\theta, \infty)$. 
Now, if $f:[0, T] \times \mathbb{R}^{n} \rightarrow \mathbb{R}$ be bounded uniformly continuous function such that $f$ is $\theta$ Hölder continuous with respect to time variables, uniformly in $x$, then $f \in C^{\theta}\left([0, T] ; X_{0}\right)$. Also $f(0, \cdot) \in C_{b}^{2 \theta}\left(\mathbb{R}^{n}\right)$ implies $f(0) \in{ }^{T_{\alpha}} D_{A}(\theta, \infty)$. Therefore, applying Theorem 4.3, we have the following result.

Theorem 4.9 Let $\theta \in\left(0, \frac{1}{2}\right)$ and $f:[0, T] \times \mathbb{R}^{n} \rightarrow \mathbb{R}$ be bounded uniformly continuous function such that $f$ is $\theta$-Hölder continuous with respect to the time variables, uniformly in $x$. Assume that $u_{0} \in C_{b}^{2+2 \theta}\left(\mathbb{R}^{n}\right)$ and $f(0, \cdot) \in C_{b}^{2 \theta}\left(\mathbb{R}^{n}\right)$. Then any mild solution, say $u$, of (4.10) is a strict solution. Moreover, ${ }^{c} D_{t}^{\alpha} u, \Delta u \in C^{\alpha \theta}\left([0, T] ; \mathrm{BUC}\left(\mathbb{R}^{n}\right)\right)$.

Again, let $f:[0, T] \times \mathbb{R}^{n} \rightarrow \mathbb{R}$ be a bounded function such that

$$
\sup _{s \neq t, x \neq y} \frac{|f(t, x)-f(s, y)|}{|s-t|^{\theta}+|x-y|^{2 \theta}}<\infty
$$

Then $f \in C^{\theta}\left([0, T] ;^{T_{\alpha}} D_{A}(\theta, \infty)\right)$. Hence, by Theorem 4.7, we have the following result.

Theorem 4.10 Let $\theta \in\left(0, \frac{1}{2}\right)$ and $f:[0, T] \times \mathbb{R}^{n} \rightarrow \mathbb{R}$ be a bounded function satisfying the condition (4.13). Assume that $u_{0} \in C_{b}^{2+2 \theta}\left(\mathbb{R}^{n}\right)$. Then any mild solution, say $u$, of (4.10) is a strict solution. Moreover, ${ }^{c} D_{t}^{\alpha} u, \Delta u \in C^{\alpha \theta}\left([0, T] ; \operatorname{BUC}\left(\mathbb{R}^{n}\right)\right)$.

\section{Conclusion}

We constructed two classes of interpolation spaces in terms of solution operators of fractional abstract Cauchy problem, and showed that these classes coincide with the real interpolation space. Moreover, it is found that these newly formulated interpolation spaces are useful to prove the strict Hölder regularity of a mild solution of a fractional ACP in the semigroup fashion as of the classical case.

\section{Acknowledgements}

The authors would like to thank the reviewers for carefully reading the manuscript and raising some insightful comments, which were really helpful to improve the manuscript.

Funding

Science and Engineering Research Board, New Delhi, India for providing support through funded project File no. MTR/2019/000437. Ministry of Human Resource Development (MHRD), GOI for extending the support of assistantship.

Availability of data and materials

Articles are cited appropriately and listed in the bibliography section.

\section{Competing interests}

The authors declare that they have no competing interests.

\section{Authors' contributions}

MMA and SD contributed in conception and design of study. They also participated in the analysis and proofs of the study. Authors made substantial contribution in drafting the manuscript. SD and DB participated in revising the manuscript critically for important intellectual content. All authors read and approved the final manuscript.

\section{Author details}

'Department of Mathematics, IIT Madras, Chennai, India. ²Department of Mathematics, Cankaya University, 06530 Balgat, Ankara, Turkey. ${ }^{3}$ Institute of Space Sciences, Magurele-Bucharest, Romania. ${ }^{4}$ Department of Medical Research, China Medical University Hospital, China Medical University, Taichung, Taiwan.

\section{Publisher's Note}

Springer Nature remains neutral with regard to jurisdictional claims in published maps and institutional affiliations. 


\section{References}

1. Lunardi, A.: Analytic Semigroups and Optimal Regularity in Parabolic Problems. Modern Birkhäuser Classics, p. 424. Springer, Basel (1995)

2. Sinestrari, E.: On the abstract Cauchy problem of parabolic type in spaces of continuous functions. J. Math. Anal. Appl. 107(1), 16-66 (1985). https://doi.org/10.1016/0022-247X(85)90353-1

3. Metzler, R., Klafter, J.: The random walk's guide to anomalous diffusion: a fractional dynamics approach. Phys. Rep. 339(1), 77 (2000). https://doi.org/10.1016/S0370-1573(00)00070-3

4. Bazhlekova, E.: The abstract Cauchy problem for the fractional evolution equation. Fract. Calc. Appl. Anal. 1(3), 255-270 (1998)

5. Wang, R.-N., Chen, D.-H., Xiao, T.-J.: Abstract fractional Cauchy problems with almost sectorial operators. J. Differ. Equ. 252(1), 202-235 (2012). https://doi.org/10.1016/j.jde.2011.08.048

6. Byszewski, L.: Theorems about the existence and uniqueness of solutions of a semilinear evolution nonlocal Cauchy problem. J. Math. Anal. Appl. 162(2), 494-505 (1991). https://doi.org/10.1016/0022-247X(91)90164-U

7. Pandey, D.N., Ujlayan, A., Bahuguna, D.: On a solution to fractional order integrodifferential equations with analytic semigroups. Nonlinear Anal. 71(9), 3690-3698 (2009). https://doi.org/10.1016/j.na.2009.02.018

8. Bahuguna, D.: Existence, uniqueness and regularity of solutions to semilinear nonlocal functional differential problems. Nonlinear Anal., Theory Methods Appl. 57(7-8), 1021-1028 (2004)

9. Dubey, S., Sharma, M.: Solutions to fractional functional differential equations with nonlocal conditions. Fract. Calc. Appl. Anal. 17(3), 654-673 (2014). https://doi.org/10.2478/s13540-014-0191-3

10. Bajlekova, E.G.: Fractional evolution equations in Banach spaces. PhD thesis, Technische Universiteit Eindhoven, The Netherlands (2001)

11. Clément, P., Gripenberg, G., Londen, S.-O.: Schauder estimates for equations with fractional derivatives. Trans. Am Math. Soc. 352(5), 2239-2260 (2000). https://doi.org/10.1090/S0002-9947-00-02507-1

12. Liu, L., Fan, Z., Li, G., Piskarev, S.: Maximal regularity for fractional Cauchy equation in Hölder space and its approximation. Comput. Methods Appl. Math. 19(4), 779-796 (2019). https://doi.org/10.1515/cmam-2018-0185

13. Chen, C., Li, M.: On fractional resolvent operator functions. Semigroup Forum 80(1), 121-142 (2010). https://doi.org/10.1007/s00233-009-9184-7

14. Guswanto, B.H.: On the properties of solution operators of fractional evolution equations. J. Fract. Calc. Appl. 6(1), 131-159(2015)

15. Pazy, A.: Semigroups of Linear Operators and Applications to Partial Differential Equations. Applied Mathematical Sciences, vol. 44, p. 279. Springer, New York (1983). https://doi.org/10.1007/978-1-4612-5561-1

16. Li, C., Li, M.: Hölder regularity for abstract fractional Cauchy problems with order in $(0,1)$. J. Appl. Math. Phys. 6 310-319 (2018)

17. Rakotondratsimba, Y.: Weighted norm inequalities for Riemann-Liouville fractional integrals of order less than one. Z. Anal. Anwend. 16(4), 801-829 (1997). https://doi.org/10.4171/ZAA/793

18. Andersen, K.F., Heinig, H.P.: Weighted norm inequalities for certain integral operators. SIAM J. Math. Anal. 14(4), 834-844 (1983). https://doi.org/10.1137/0514064

19. Hardy, G.H., Littlewood, J.E., Pólya, G.: Inequalities, 2nd edn. Cambridge University Press, Cambridge (1952)

20. Lunardi, A.: Interpolation Theory. Appunti. Scuola Normale Superiore di Pisa, 3rd edn. (Nuova Serie) [Lecture Notes. Scuola Normale Superiore di Pisa (New Series)], vol. 16, p. 199. Edizioni della Normale, Pisa (2018). https://doi.org/10.1007/978-88-7642-638-4

21. Li, F.-B., Li, M.: On maximal regularity and semivariation of $\alpha$-times resolvent families. Adv. Pure Math. 3, 680-684 (2013)

\section{Submit your manuscript to a SpringerOpen ${ }^{\circ}$ journal and benefit from:}

- Convenient online submission

- Rigorous peer review

- Open access: articles freely available online

- High visibility within the field

- Retaining the copyright to your article

Submit your next manuscript at $>$ springeropen.com 